\title{
Cognitive Impairment after Chemotherapy Related to Atypical Network Architecture for Executive Control
}

\author{
Jay F. Piccirillo ${ }^{a, b}$ Frances Mei Hardin ${ }^{b}$ Joyce Nicklaus ${ }^{b}$ Dorina Kallogjeri ${ }^{b}$ \\ Michael Wilson $^{b}$ Cynthia X. Ma ${ }^{a, g}$ Rebecca S. Coalson ${ }^{c, d}$ Joshua Shimony ${ }^{d}$ \\ Bradley L. Schlaggar ${ }^{c-f}$ \\ ${ }^{a}$ Alvin J. Siteman Cancer Center at Barnes-Jewish Hospital and Washington University School of Medicine in \\ St. Louis, and Departments of ${ }^{\mathrm{b}}$ Otolaryngology - Head and Neck Surgery, ${ }^{\mathrm{C}}$ Neurology, ${ }^{\mathrm{d}}$ Radiology, ${ }^{\mathrm{e}}$ Pediatrics and \\ ${ }^{\mathrm{f}}$ Anatomy and Neurobiology, and ${ }^{9}$ Division of Medical Oncology, Department of Medicine, Washington University \\ School of Medicine in St. Louis, St. Louis, Mo., USA
}

\section{Key Words}

Cognitive disorders · Diagnostic imaging · Neuroimaging ·

Breast neoplasms · Chemotherapy · Complications

\begin{abstract}
Objectives: A common complaint of cancer patients is the experience of cognitive difficulty during and after chemotherapy. We hypothesized that cognitive impairment may result from dysfunction in large-scale brain networks, particularly those involved in attentional control. Methods: Using a case-control design, this study includes women with a history of invasive ductal or lobular triple-negative breast cancer who completed standard adjuvant chemotherapy within 2 years of study entry. Women who reported cognitive impairment by the Global Rating of Cognition question were considered to be cases $(n=15)$. Women who reported no cognitive impairment were considered to be controls $(n=13)$. All enrolled participants were eligible for MRI investigation and underwent resting-state functional connectivity MRI. Results: Women who self-reported cognitive impairment were found to have disrupted resting-state functional connectivity, as measured by MRI, when compared to women who did not self-report cognitive impairment. These find-
\end{abstract}

ings suggest that some women may be more sensitive to the standard treatments for breast cancer and that this increased sensitivity may result in functional connectivity alterations in the brain networks supporting attention and executive function. Conclusions: Neuroimaging analyses confirmed selfreported cognitive deficits in women with breast cancer treated with chemotherapy.

(c) 2015 S. Karger AG, Basel

\section{Introduction}

Chemotherapy-associated cognitive impairment (CACI), or 'chemobrain', is a phenomenon in which a subset of cancer survivors suffers cognitive dysfunction after chemotherapy. A recently published report from the International Cognition and Cancer Task Force (ICCTF) [1] concluded that 'neuropsychological studies have shown cognitive dysfunction in $13-70 \%$ of patients receiving chemotherapy'. This cognitive impairment manifests in a variety of ways, most notably as a memory im-

Presented at the 2013 San Antonio Breast Cancer Symposium, San Antonio, Tex., USA, December 2013.

\section{KARGER 125}

(c) 2015 S. Karger AG, Base

0030-2414/15/0886-0360\$39.50/0

E-Mail karger@karger.com

www.karger.com/ocl
Prof. Jay F. Piccirillo

Division of Medical Oncology, Department of Otolaryngology - Head and Neck Surgery Washington University School of Medicine in St. Louis, 660 South Euclid Av. Campus Box 8115, St. Louis, MO 63110 (USA)

E-Mail piccirilloj@ent.wustl.edu 
pairment, a decreased capability to perform executive functions including working memory, and deficits in psychomotor and processing speed [2]. For many patients, cognitive impairment proves to be debilitating as it negatively impacts the quality of life and hinders occupational goals [1]. Nelson and Suls [2] recently published a review of the literature on the relationship between chemotherapy and cognitive impairment and observed a similarly wide range of estimates for the incidence of $\mathrm{CACI}$ as cited by the ICCTF. They concluded that this wide estimate for CACI from various studies could be attributed to a variety of reasons, including methodological differences in their assessment of cognitive function, and they suggested that new research approaches were needed to study chemotherapy-associated cognitive changes.

Implementing advanced neuroimaging techniques to investigate CACI constitutes a promising complement to self-reports and neuropsychological assessments, both of which are not capable of revealing the neural mechanisms underlying CACI. Resting-state functional connectivity MRI (rs-fcMRI) is used to infer functional relatedness between various regions of the brain [3]. Since cognition relies on synergistic activities of large neural populations organized by function, a network-based analytical approach is useful for understanding the underlying neurobiological mechanisms of cognitive deficits [4]. rs-fcMRI has previously been used to define network disruptions resulting in the cognitive deficits associated with various pathologic conditions, including - but not limited to - Alzheimer's disease [5], stroke [6], and depression [7]. Bruno et al. [8] used rs-fcMRI and graph theoretical analysis to examine the functional connectivity in breast cancer survivors treated with chemotherapy relative to healthy women. Compared to the healthy controls, the breast cancer group displayed an altered global brain network organization characterized by significantly decreased global clustering as well as disrupted network characteristics in frontal, striatal, and temporal regions. These authors suggest that this pattern of altered network organization is likely to result in a reduced efficiency of information transfer. The same group [9], using rs-fcMRI, found that disrupted default mode network (DMN) connectivity among breast cancer patients who received chemotherapy may explain longterm cognitive difficulties. Studies using structural MRI have revealed decreased gray matter volumes in frontal, temporal, and cerebellar regions in breast cancer patients following a course of adjuvant chemotherapy [10]. Based on rs-fcMRI, other studies suggested alterations in the functional network architecture of the brains of women with breast cancer who received chemotherapy, leading to decreased network efficiency and implicated brain systems important for executive function [8]. In their review of the literature, O'Farrell et al. [10] cited several studies investigating the effects of CACI and found increased activation in the prefrontal cortex and cerebellum. The authors conclude that these findings might represent a compensatory mechanism following a decrease in cognitive ability and that pretreatment baseline assessments are necessary to reveal changes in brain integrity resulting from the neurotoxic effects of chemotherapy. In contrast to these studies, the present study investigates a population of women whose breast cancer has been treated with chemotherapy, using self-reports to delineate subgroups of women who do or do not describe cognitive impairment.

Based on the contention that systemic chemotherapy causes functional disruptions in several neural systems, most notably the networks responsible for attention and executive control [11,12], our focus in the current study is on the frontoparietal attention network $[13,14]$ (composed of the precuneus and the bilateral inferior parietal and dorsolateral prefrontal cortices) and the cingulo-opercular control network (composed of the frontal operculum and the bilateral medial frontal, midcingulate, and right supramarginal gyri). The frontoparietal network is associated with moment-to-moment top-down task control [13] and is flexibly supporting goal-oriented processes [15]. The cingulo-opercular network is associated with the stable maintenance of overall task configurations. These functions are consistent with the broad range of deficits subjectively reported by many patients after chemotherapy $[11,12,16]$. A novel aspect of this study is the use of self-reports rather than results from neurocognitive testing to define subgroups of patients with and without complaints of CACI.

\section{Subjects and Methods}

\section{Design and Setting}

This was a case-control study of female breast cancer survivors who received chemotherapy as part of their cancer treatment. The impaired cohort (i.e., cases) was defined as women who affirmed cognitive impairment, and the nonimpaired cohort (i.e., controls) was defined as women who did not affirm cognitive impairment. Approval by the Human Research Protection Office, Washington University in St. Louis, was obtained prior to recruitment.

\section{Participants}

The recruited participants were between the ages of 35 and 70 years, had been diagnosed with invasive ductal or lobular breast cancer at stage I, II, or III [American Joint Committee on Cancer (AJCC) Staging Manual, ed 7, 2010] within the previous 2 years, and had finished chemotherapy treatment at least 30 days prior to participation. Participants could be pre- or postmenopausal with 
early-stage breast cancer, receiving standard adjuvant chemotherapy including anthracycline and/or taxane. The exclusion criteria included (1) evidence of other active cancers within the previous year, (2) receipt of skull-base radiation treatment within the previous year, or (3) a history of brain trauma or disease.

The participants completed the following assessment forms: (1) medical history and health information, (2) Cognitive Failures Questionnaire (CFQ) [17], and (3) Global Rating of Cognition (GRC). The CFQ is a validated self-report questionnaire that contains 25 items and measures failures in perception, memory, and motor function. The GRC is a single-item self-report question that uses a Likert-type scale to rate the impact of cognitive impairments on daily life. Based upon responses to the GRC question, the subjects were assigned to the 'impaired' or the 'nonimpaired' group. There were 15 subjects who endorsed a GRC response of 'extremely affected', 'strongly affected', or 'moderately affected' by their impairment and were classified as impaired. There were 13 subjects who endorsed a GRC response of 'slightly affected' or 'not affected' and were classified as nonimpaired.

\section{Neuroimaging Data Collection}

Scans were performed on a Siemens 3T Tim Trio MRI scanner at Washington University in St. Louis. rs-fcMR and anatomical images were collected during the same imaging session. An asymmetric spin-echo echo-planar imaging $(\mathrm{EPI})$ pulse sequence $(\mathrm{TR}=$ $2,200 \mathrm{~ms} ; \mathrm{TE}=27 \mathrm{~ms}$; flip angle $=90^{\circ} ; 4 \times 4 \times 4 \mathrm{~mm}$ voxels $)$ captured images of blood oxygenation level-dependent (BOLD) contrast responses $[18,19]$. The EP images of the whole brain involved volume acquisitions across 36 odd-even, contiguously interleaved, bicommissurally aligned axial slices. A T1-weighted, structural magnetization-prepared rapid acquisition gradient echo (MPRAGE) image was acquired across 176 sagittal slices $(\mathrm{TR}=2,400$ $\mathrm{ms}$; TE $=3.09 \mathrm{~ms}$; flip angle $=8^{\circ}$; inversion time $=1,000 \mathrm{~ms} ; 1 \times 1$ $\times 1 \mathrm{~mm}$ voxels). Additionally, a T2-weighted structural image obtained across 36 axial slices ( $\mathrm{TR}=6,150 \mathrm{~ms}$; $\mathrm{TE}=86 \mathrm{~ms}$; flip angle $=120^{\circ} ; 1 \times 1 \times 4 \mathrm{~mm}$ voxels) was in register with the EPI and aided alignment between axial EP and sagittal MP-RAGE image slices [20]. Three 164-frame (6-min) EPI runs recorded spontaneous brain activity while the participants were awake, performed no task, and remained with their eyes closed in a darkened room. Data on 3 runs, 6 min each, were collected so that (1) there would be enough data remaining after removing frames with motion, (2) we had available a sufficient representation of the lowest frequencies of spontaneous BOLD signal fluctuations for rs-fcMRI analysis, and (3) a single 18-min run could be avoided, which would have been demanding on patients as they were asked to hold still and not to fall asleep. This strategy of concatenating BOLD volumes for rs-fcMRI is common and has been adopted in multiple studies [21-24] as well as the in Human Connectome Project [23].

\section{Image Preprocessing}

EP image preprocessing started with compensation for systematic slice-dependent differences from interleaved odd-even slice acquisition and alignment of the time for each slice to the beginning of each volume acquisition using sinc interpolation. Next, corrections for intensity differences within runs utilized a wholebrain mean signal intensity normalized to mode 1,000 . These timeand intensity-adjusted slices were realigned within and across runs using rigid-body correction for interframe head motions [25-27]. The across-run-realigned slices were resampled to $3-\mathrm{mm}^{3}$ voxels and registered to an atlas template by computing 12-parameter affine transformations between an average from the first frames of each EPI run and the atlas template using the individual's T2 and MP-RAGE images as intermediaries [20]. This atlas template was created using MP-RAGE structural images from 12 normal, middle-aged individuals (mean: 48 years; SD: 10.7) and registered to the Talairach atlas space $[28,29]$ based on spatial normalization methods [30].

Additional resting-state preprocessing steps were applied in MATLAB (2007a; MathWorks, Natick, Mass., USA) to reduce noise from sources unlikely to reflect neural activity [31]. These steps include demeaning and detrending each BOLD run, temporal filtering with a bandpass filter to remove frequencies $>0.009$ and $<0.08 \mathrm{~Hz}$, and spatial smoothing with a 6 -mm full-width-athalf-maximum Gaussian kernel. The BOLD signal modifications per voxel removed (through linear regression) 24 motion-related and 6 tissue-related sources of nuisance variance. The motion regressors were the 6 previously computed linear corrections for head movement, their squares, and the same for the immediately preceding time point, as derived by Volterra expansion [32]. The tissue-related regressors were a global whole-brain signal averaged over all voxels in the brain, signals in the ventricles and white matter, and their associated temporal derivatives [32]. A standard mask was used for the whole brain, ventricles, and white matter in each subject $[25,27,33,34]$.

We applied a volume censoring method [31] which removed frames of data with either $>0.4 \mathrm{~mm}$ of frame-by-frame displacement or $>4$ of the quality control parameter dvars, which measures the rate of change in signal intensity across the entire brain at each frame [35]. No additional frames before or after the censored frames were removed, and interpolation was not performed [36]. Spatial smoothing and temporal filtering as well as nuisance variable regression were repeated on the original preprocessed data, leaving out the censored frames. Since these parameters were more lenient than recommended by Power et al. [31], we plotted correlation between region pairs versus the distance between regions to check for the distance-dependent artifacts often caused by even submillimeter head motion [31,37]. Our rs-fcMRI preprocessing and processing approaches are based on methods we and others have developed over the previous 5-7 years [4, 31, 38-40].

Resting-State Analysis of Correlations between Control Regions A total of 25 frontoparietal and 14 cingulo-opercular cognitive control regions of Dosenbach et al. [13] were created by placing a 10-mm-diameter sphere around the reported coordinates (fig. 1).

A time series of BOLD signal intensity was calculated in each of the 39 regions for each subject, and within-subject Pearson correlation coefficients $(R)$ were calculated between each pair of these regions, after which the Fisher $\mathrm{R}-\mathrm{Z}$ transformation was performed [41]. For example, a $39 \times 39$ correlation matrix was generated for each subject, with each cell containing the Fisher Z-transformed Pearson R for each pair-wise correlation. Student's t tests (twotailed independent-samples t tests assuming equal variance) were calculated in MATLAB between the matrices for impaired and nonimpaired subjects for each region pair. Bonferroni's correction was used to correct for multiple comparisons. A p value $<6.75 \times$ $10^{-5}$ was considered significant. Simple linear regression was used to explore the relationship between the behavioral scores (GRC/ $\mathrm{CFQ}$ ) and the Fisher Z-standardized R correlation values (1 value per subject) in the reported connection. 


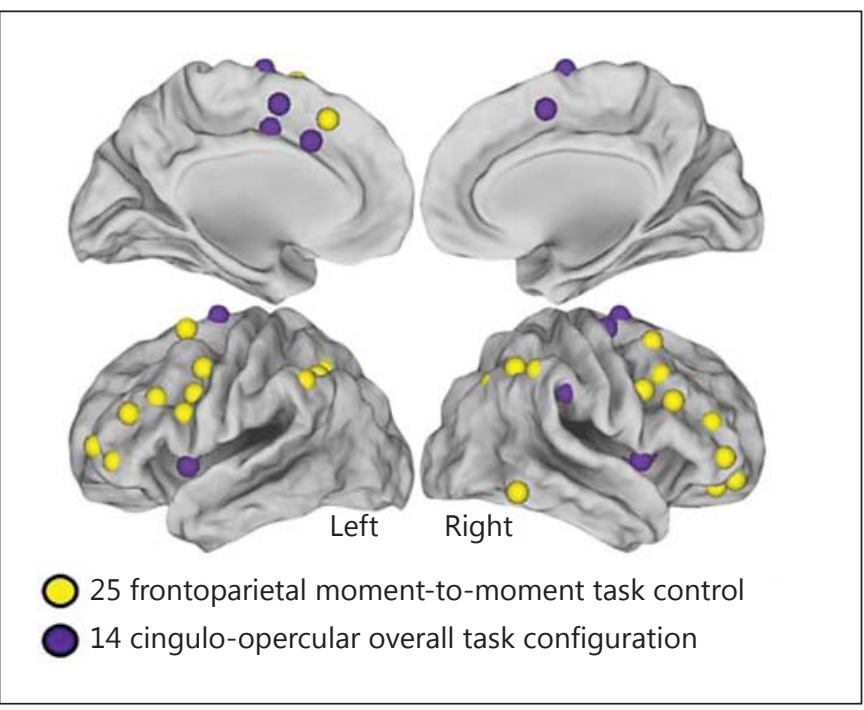

Fig. 1. A total of 39 task control regions were tested for functional connection differences between impaired and nonimpaired patients. The images show the 25 frontoparietal and 14 cingulo-opercular regions used. Note that the spheres are shown larger than the actual 10-mm-diameter size.

\section{Results}

\section{Participants}

The study population consisted of 28 females with a median age of 53 years (range: $36-69$ ). Of the 28 participants, $20(71 \%)$ were postmenopausal at the time of data acquisition. There was no significant difference between cases and controls in the distribution of menopausal status, tumor stage, the type of chemotherapy treatment received, or the presence of other comorbid ailments. The percentage of women receiving hormonal therapy in the nonimpaired control group $(67 \% ; n=8)$ was significantly greater than the percentage of women receiving hormonal therapy in the impaired case group $(27 \% ; n=4)$. Additional participant characteristics are shown in table 1 . The two groups (impaired and nonimpaired) differed in self-reported cognitive impairment as measured by the GRC (used to define the groups) as well as on the CFQ.

\section{Resting-State Functional Connectivity Results}

All 28 participants had sufficient usable rs-fcMRI data after motion censoring; the smallest number of usable frames was 138, and the largest was 476 . There was no difference between the impaired and the nonimpaired group in the number of frames kept after motion censoring [im- paired group: mean 396 (SD 89); nonimpaired group: 390 (104); $t$ test $p=0.87]$, in root mean squared movement on each run [impaired group: $0.203 \mathrm{~mm}$ (0.05); nonimpaired group: $0.197 \mathrm{~mm}(0.06)$; t test $\mathrm{p}=0.80$ ], or in the quality control measure dvars [impaired group: 1.44 (0.25); nonimpaired group: $1.55(0.24)$; t test $\mathrm{p}=0.26]$. All usable frames were included for each subject.

A significant difference $\left(\mathrm{p}=1.4 \times 10^{-5}\right)$ in connection strength between impaired (average $\mathrm{R}=0.118$ ) and nonimpaired subjects (average $R=0.346$ ) was identified between 2 regions of the frontoparietal system: the impaired subjects showed weaker functional connectivity. The functional connection between the left frontoparietal region (Talairach coordinates: $\mathrm{x}=-41, \mathrm{y}=20, \mathrm{z}=31$ ) and a right parietal region $(x=41, y=-55, z=45)$ is shown in figure 2.

Further evidence that this functional connection may be atypical in the setting of CACI is the relationship between an individual's functional connection strength and her scores on both of the behavioral measures (CFQ and GRC) (fig. 3). The subject groups were defined by the GRC. Thus, we assessed whether the correlation strength for this particular functional connection related to the GRC scores only within groups. A simple linear regression was performed between the behavioral scores and the Fisher Z-standardized R values for the reported connection ( 1 value per subject). Within the impaired group, the correlation strength had a negative relationship to the GRC, with severely affected individuals (highest GRC scores) having the lowest functional connection strength and $31 \%$ of the variance explained $(p=0.031)$. By contrast, within the nonimpaired group, there was a nonsignificant relationship between connection strength and GRC score $(\mathrm{p}=0.34)$.

We also tested the relationship between CFQ score and correlation strength across both groups (fig. 4). The negative correlation explains $31 \%$ of the variance $(\mathrm{p}=$ 0.0022 ). By contrast, no such relationship was determined within either the impaired $(\mathrm{p}=0.89)$ or the nonimpaired group ( $\mathrm{p}=0.65)$.

To assess the impact of subject motion, we examined the present data based on both the $39 \times 39$ correlation matrix and a larger matrix generated using a set of 264 regions [31] that includes those 39 regions and has a broader coverage over the cerebrum. Importantly, no distance-dependent artifacts were found for either region set, indicating that the choices made for preprocessing adequately removed the potential contaminating effect of motion artifacts from these data. 
Table 1. Description of the study population

\begin{tabular}{|c|c|c|c|c|}
\hline Demographics & $\begin{array}{l}\text { Total } \\
(\mathrm{n}=28)\end{array}$ & $\begin{array}{l}\text { Impaired } \\
(\mathrm{n}=15)\end{array}$ & $\begin{array}{l}\text { Nonimpaired } \\
(\mathrm{n}=13)\end{array}$ & $\mathrm{p}$ value $\mathrm{a}^{\mathrm{a}}$ \\
\hline Age, years & $53(36-69)$ & $54(36-69)$ & $52(40-67)$ & 0.555 \\
\hline \multicolumn{5}{|l|}{ Race } \\
\hline White & $18(64 \%)$ & $9(60 \%)$ & $9(69 \%)$ & \multirow[t]{3}{*}{0.596} \\
\hline Black & $8(29 \%)$ & $4(27 \%)$ & $4(31 \%)$ & \\
\hline Asian & $2(7 \%)$ & $2(13 \%)$ & 0 & \\
\hline \multicolumn{5}{|l|}{ Employment } \\
\hline Full-time & $14(50 \%)$ & $6(40 \%)$ & $8(62 \%)$ & \multirow[t]{5}{*}{0.464} \\
\hline Part-time & $6(21 \%)$ & $4(26 \%)$ & $2(15 \%)$ & \\
\hline Unemployed & $3(11 \%)$ & $1(7 \%)$ & $2(15 \%)$ & \\
\hline Retired & $3(11 \%)$ & $3(20 \%)$ & 0 & \\
\hline Other & $2(7 \%)$ & $1(7 \%)$ & $1(8 \%)$ & \\
\hline \multicolumn{5}{|l|}{ Education } \\
\hline High school/GED equivalent & $8(29 \%)$ & $6(40 \%)$ & $2(15 \%)$ & \multirow[t]{5}{*}{0.202} \\
\hline Associate degree or some college & $7(25 \%)$ & $4(27 \%)$ & $3(24 \%)$ & \\
\hline Bachelor's degree & $5(18 \%)$ & $1(6 \%)$ & $4(31 \%)$ & \\
\hline Master's degree & $6(21 \%)$ & $4(27 \%)$ & $2(15 \%)$ & \\
\hline $\mathrm{PhD}, \mathrm{MD}$, JD, or other higher degree & $2(7 \%)$ & 0 & $2(15 \%)$ & \\
\hline CFQ score & $47(12-81)$ & $57(46-81)$ & $28(12-56)$ & $<0.001$ \\
\hline \multicolumn{5}{|l|}{ GRC } \\
\hline Not at all cognitively impaired & $6(21 \%)$ & 0 & $6(46 \%)$ & \multirow[t]{5}{*}{$<0.001$} \\
\hline Slightly cognitively impaired & $7(25 \%)$ & 0 & $7(54 \%)$ & \\
\hline Moderately cognitively impaired & $5(18 \%)$ & $5(33 \%)$ & 0 & \\
\hline Strongly cognitively impaired & $7(25 \%)$ & $7(47 \%)$ & 0 & \\
\hline Extremely cognitively impaired & $3(11 \%)$ & $3(20 \%)$ & 0 & \\
\hline
\end{tabular}

Values for age and CFQ are denoted as medians (min-max). GED = General Educational Development. ${ }^{\mathrm{a}}$ The Mann-Whitney test was used for continuous variables, and Fisher's exact test was used for categorical variables.

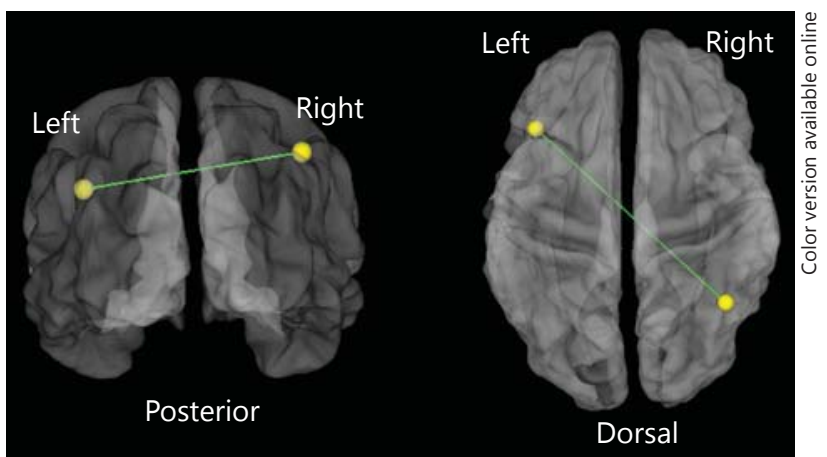

Fig. 2. A functional connection in the frontoparietal system shows reliable differences between impaired and nonimpaired patients. Student's t tests between impaired and nonimpaired patients resulted in a single functional connection between frontoparietal regions with a correlation strength difference (more correlated in nonimpaired than in impaired patients) that meets the stringent Bonferroni correction for multiple comparisons. The left frontal (Talairach coordinates: $x=-41, y=20, z=31)$ to right parietal $(x=41, y=-55$, $\mathrm{z}=45$ ) connection, shown on a translucent brain in 2 views (left panel: posterior; right panel: dorsal) had a mean standardized $\mathrm{R}$ of 0.346 for nonimpaired and 0.118 for impaired patients $(\mathrm{p}=0.00001)$.

\section{Discussion}

The findings from this case-control cross-sectional study of cognitive impairment in female breast cancer patients demonstrate that women who self-reported cognitive impairment were found to have disrupted functional connectivity within brain networks implicated in cognitive control. In addition, the disrupted functional connection that was identified indexed the extent of cognitive impairment within the group reporting impairment. These findings suggest that the standard therapeutic levels of chemotherapy for some breast cancer patients may result in altered functional connectivity in the brain networks supporting attention and executive function. This effect, in turn, may contribute to the self-reported cognitive difficulties after receiving chemotherapy among a subset of breast cancer patients.

We hypothesized that the executive dysfunction described by women with chemotherapy-induced cognitive impairment would localize to brain systems critical for 


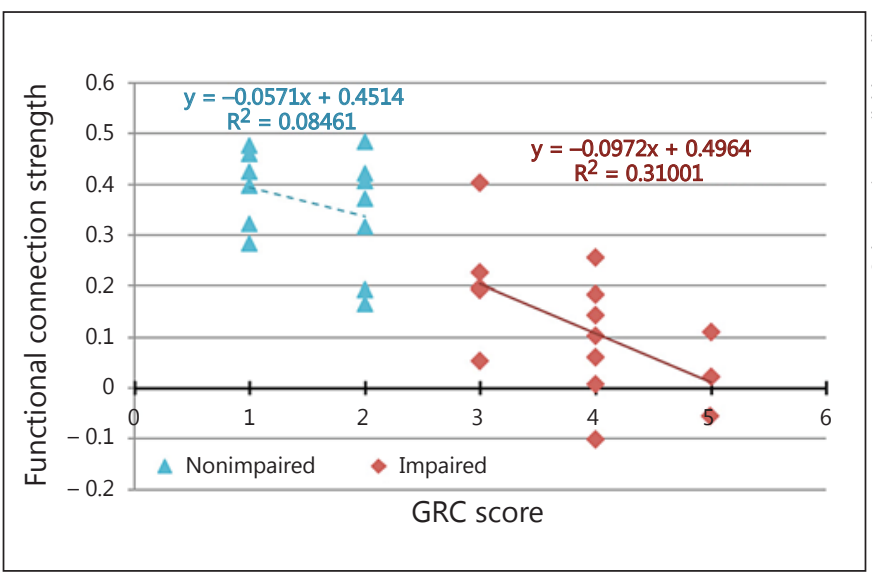

Fig. 3. Correlation values (standardized R) for the functional connection shown in figure 2 plotted against the self-reported measure of severity of cognitive impairment GRC. The functional connection strength shows a robust correlation with cognitive impairment for the impaired but not for the nonimpaired patients.

executive or 'top-down' control. Specifically, building upon our 'dual networks architecture for top-down control' [13], where a distinct frontoparietal system oversees rapid, adaptive online control and a separate cinguloopercular system oversees stable, resilient task set maintenance, we hypothesized that there would be disrupted resting-state functional connectivity within these now well-defined systems. The primary result from the functional connectivity data (i.e., that a single functional connection between 2 regions within the frontoparietal system shows a robust difference in strength between chemotherapy-treated breast cancer survivors who describe CACI and those who do not) is partially consistent with this hypothesis. In addition, the observation that the strength of this functional connectivity seems to provide an index of the perceived severity of impairment with the group experiencing impairment lends plausibility to this relationship.

The majority of published studies that have investigated CACI have only used batteries of standard neurocognitive tests. The ICCTF [1] defines impairment as scoring 1.5 SDs below average on 1 or more standard neurocognitive assessments. Unfortunately, this definition reflects a population-level pre-post function difference rather than an individual difference. In addition, the use of neuropsychological tests is problematic, since these tests are subject to practice effects and do not fully describe the extent of cognitive impairment $[16,42]$. Pa-

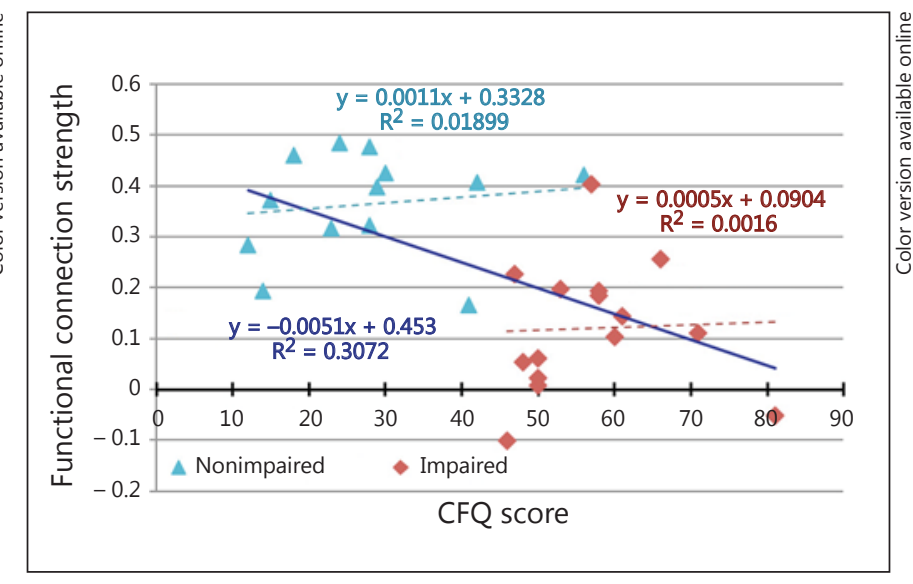

Fig. 4. Correlation values for the functional connection shown in figure 2 plotted against the CFQ score. Higher values indicate greater perceived cognitive impairment. There is a robust negative correlation across all individuals with a higher functional connection strength associated with lower perceived impairment. However, no significant relationship was detected within either group alone.

tients may report difficulty in performing mental tasks while simultaneously scoring within a normal range of cognitive function. Self-reported measures of cognition are more sensitive in detecting subtle cognitive changes that may be functionally relevant to the patient [2].

Previous neuroimaging research has demonstrated abnormalities in brain structure after chemotherapy among breast cancer patients [43-45]. For example, Deprez and colleagues $[46,47]$ used diffusion tensor imaging to study the cerebral white matter integrity in women with breast cancer who received chemotherapy. Compared to controls, the breast cancer patients showed decreased fractional anisotropy (FA) in frontal and temporal white matter tracts and increased mean diffusivity in frontal white matter. Reduced FA is typically interpreted to reflect reduced white matter integrity. An analysis of the study's results showed a significant correlation between FA and performance on standard neuropsychological tests. In a subsequent, nested case-control study, there were significant decreases in FA in breast cancer patients after exposure to chemotherapy. In addition, performance changes in attention and verbal memory correlated with mean regional FA changes. The authors concluded that concurrent longitudinal changes in white matter integrity and cognition were observed after chemotherapy treatment.

The frontoparietal system is thought to be important for the initiation and rapid adjustment of control during 
the carrying out of attention-demanding tasks [13]. Future work should further test the hypothesis that this system is differentially affected in patients with CACI. For example, it would be helpful to relate the strength of functional connectivity to psychometric measures of executive control. In addition, task-based fMRI, using tasks designed to address rapid, adaptive online control, could be helpful in investigating the relationship discerned based on resting-state functional connectivity data.

Bruno et al. [8] demonstrated in 2012 that the functional network architecture of the brains of women with breast cancer who received chemotherapy differed in standard network metrics from that of the brains of women who did not receive chemotherapy. The findings based on rs-fcMRI and graph theory-based approaches suggested alterations leading to decreased network efficiency and implicated brain systems important for executive function. Subsequent work, done in collaboration with Kesler et al. [9], demonstrated that by using machine learning and a multivariate pattern classification approach for rsfcMRI data on the DMN, it was possible to classify single individuals as belonging to a healthy control group or either a chemotherapy-treated or a non-chemotherapytreated breast cancer survivor group. In follow-up work, implementing the same machine learning pattern classification approach, but this time using task-based fMRI from an attention demanding task for functional connectivity, Hosseini and Kesler [48] demonstrated a comparably robust capacity to classify the same set of individuals accurately as belonging to either healthy controls or chemotherapy-treated or non-chemotherapy-treated patients with breast cancer. A successful classification appeared to place the greatest weight on brain regions in the frontal and parietal cortices. Findings by McDonald et al. [11] showed a decreased frontal gray matter density after chemotherapy, which was accompanied by self-reported difficulties in executive function. Kesler et al. [12] found significantly reduced activation in the left middle dorsolateral prefrontal cortex and the premotor cortex in breast cancer survivors compared with healthy controls. Breast cancer survivors who received chemotherapy demonstrated significantly reduced left caudal lateral prefrontal cortex activation as well as increased perseverative errors and reduced processing speed. Finally, Kesler et al. [9] concluded that a disrupted DMN connectivity may help explain long-term cognitive difficulties following chemotherapy in breast cancer patients.

Taken together, these observations implicate alterations in the overall functional network architecture in the brains of chemotherapy-treated breast cancer patients and fit the notion that chemotherapeutic effects on cognition are unlikely to be restricted to a specific region or set of regions. Thus, we do not believe that a single functional connection is sufficient as an explanation for the pathobiology associated with chemotherapy-associated cognitive changes. Along those lines, a multivariate pattern classification in the form implemented by Hosseinni and Kesler [48] has the substantial likelihood of providing an additional capacity to demonstrate CACI effects beyond that available by standard univariate analyses. While the above-mentioned studies were designed to address the question of how chemotherapy in the setting of breast cancer influences the brain's functional network architecture, our study was designed to address the question of which brain systems are altered differentially in patients who, in the setting of chemotherapy, report cognitive deficits. A larger sample size than that used in the current study may make it possible to discern additional group differences.

\section{Limitations}

The present study has several limitations. First, the numbers of patients with and without self-reported cognitive impairment are relatively small. The low power produces an increased risk of both type I and type II errors. While stringent multiple comparison correction methods were implemented to reduce the likelihood of type II errors, there remains a concern for both spurious false-positive and false-negative findings. Thus, the results reported in the present study must be considered those of a pilot investigation. Second, the percentage of the women receiving hormonal therapy was significantly different between the two groups. We believe this finding is spurious and does not reflect a true biological effect or diminish the findings of disrupted functional connectivity among women who received chemotherapy. To explore the role of hormonal therapy in breast cancer women with CACI, a larger study should be conducted. Third, as the women received a large number of chemotherapeutic agents, we were unable to explore whether specific agents are more likely to cause CACI and changes in functional connectivity.

\section{Clinical Implications}

The clinical relevance of the results from this pilot investigation is noteworthy. Understanding the brain systems implicated in CACI has a substantial likelihood of shaping rational interventions - both pharmacotherapeutic and cognitive/behavioral ones. In addition, having neuroimaging biomarkers for CACI, in combination 
with increasingly sophisticated analysis strategies such as multivariate pattern analysis, increases the possibility that we will be able to predict which patient with breast cancer is most likely to suffer from CACI. That sort of predictive power could lead to alterations in chemotherapeutic regimens, the implementation of preemptive interventions, or other approaches to mitigate cognitive burdens.

\section{Acknowledgements}

This study was supported, in part, by grants from the Foundation for Barnes-Jewish Hospital-Cancer Frontier Fund (7540-55), the NIH Clinical and Translational Science Award (CTSA; UL1 TR000448), and the Intellectual and Developmental Disabilities
Research Center at Washington University in St. Louis (NIH/ NICHD P30 HD062171). The ClincialTrials.gov No. is NCT01578083.

In our research, the authors used REDCap for data collection and storage, and this part was supported by CTSA Grant UL1 TR000448 as well as the Siteman Comprehensive Cancer Center and NCI Cancer Center Support Grant P30 CA091842.

We acknowledge the assistance of Caroline Bumb (MHS) in the identification and recruitment of study subjects; of Timothy Wolf (OTD, MSCI, OTR/L) for intellectual input regarding measuring and reporting results of cognitive impairment in the study protocol and manuscript, and of Jonathan Peelle (PhD) for his contribution to the interpretation of the data and critical review of the manuscript.

The studies presented in this work were conducted using the scanning and special services in the MIR Center for Clinical Imaging Research located at the Washington University Medical Center.

\section{References}

1 Wefel JS, Vardy J, Ahles T, Schagen SB: International Cognition and Cancer Task Force recommendations to harmonise studies of cognitive function in patients with cancer. Lancet Oncol 2011;12:703-708.

2 Nelson WL, Suls J: New approaches to understand cognitive changes associated with chemotherapy for non-central nervous system tumors. J Pain Symptom Manage 2013;46: 707-721.

-3 Zhang D, Raichle ME: Disease and the brain's dark energy. Nat Rev Neurol 2010;6:15-28.

-4 Power JD, Schlaggar BL, Lessov-Schlaggar $\mathrm{CN}$, Petersen SE: Evidence for hubs in human functional brain networks. Neuron 2013;79: 798-813.

5 Greicius MD, Srivastava G, Reiss AL, Menon V: Default-mode network activity distinguishes Alzheimer's disease from healthy aging: evidence from functional MRI. Proc Natl Acad Sci USA 2004;101:4637-4642.

6 Corbetta M, Tansy AP, Stanley CM, Astafiev SV, Snyder AZ, Shulman GL: A functional MRI study of preparatory signals for spatial location and objects. Neuropsychologia 2005; 43:2041-2056.

7 Sheline YI, Barch DM, Price JL, Rundle MM, Vaishnavi SN, Snyder AZ, Mintun MA, Wang S, Coalson RS, Raichle ME: The default mode network and self-referential processes in depression. Proc Natl Acad Sci USA 2009;106: 1942-1947.

8 Bruno J, Hosseini SM, Kesler S: Altered resting state functional brain network topology in chemotherapy-treated breast cancer survivors. Neurobiol Dis 2012;48:329-338.

$\checkmark 9$ Kesler SR, Wefel JS, Hosseini SM, Cheung M, Watson CL, Hoeft F: Default mode network connectivity distinguishes chemotherapytreated breast cancer survivors from controls. Proc Natl Acad Sci USA 2013;110:1160011605.
10 O’Farrell E, MacKenzie J, Collins B: Clearing the air: a review of our current understanding of 'chemo fog'. Curr Oncol Rep 2013;15:260269.

11 McDonald BC, Conroy SK, Smith DJ, West JD, Saykin AJ: Frontal gray matter reduction after breast cancer chemotherapy and association with executive symptoms: a replication and extension study. Brain Behav Immun 2013;30(suppl):S117-S125.

12 Kesler SR, Kent JS, O’Hara R: Prefrontal cortex and executive function impairments in primary breast cancer. Arch Neurol 2011;68: 1447-1453.

13 Dosenbach NU, Fair DA, Cohen AL, Schlaggar BL, Petersen SE: A dual-networks architecture of top-down control. Trends Cogn Sci 2008;12:99-105.

14 Petersen SE, Posner MI: The attention system of the human brain: 20 years after. Annu Rev Neurosci 2012;35:73-89.

15 Duncan J: The multiple-demand (MD) system of the primate brain: mental programs for intelligent behaviour. Trends Cogn Sci 2010; 14:172-179.

16 Tannock IF, Ahles TA, Ganz PA, Van Dam FS: Cognitive impairment associated with chemotherapy for cancer: report of a workshop. J Clin Oncol 2004;22:2233-2239.

-17 Broadbent DE, Cooper PF, Fitzgerald P, Parkes KR: The Cognitive Failures Questionnaire (CFQ) and its correlates. Br J Clin Psychol 1982;21(pt 1):1-16.

18 Kwong KK, Belliveau JW, Chesler DA, Goldberg IE, Weisskoff RM, Poncelet BP, Kennedy DN, Hoppel BE, Cohen MS, Turner R: Dynamic magnetic resonance imaging of human brain activity during primary sensory stimulation. Proc Natl Acad Sci USA 1992;89:56755679.
19 Ogawa S, Tank DW, Menon R, Ellermann JM, Kim SG, Merkle H, Ugurbil K: Intrinsic signal changes accompanying sensory stimulation: functional brain mapping with magnetic resonance imaging. Proc Natl Acad Sci USA 1992;89:5951-5955.

20 Ojemann JG, Akbudak E, Snyder AZ, McKinstry RC, Raichle ME, Conturo TE: Anatomic localization and quantitative analysis of gradient refocused echo-planar fMRI susceptibility artifacts. Neuroimage 1997;6:156-167.

21 Luking KR, Repovs G, Belden AC, Gaffrey MS, Botteron KN, Luby JL, Barch DM: Functional connectivity of the amygdala in earlychildhood-onset depression. J Am Acad Child Adolesc Psychiatry 2011;50:1027-1041.

22 Gaffrey MS, Luby JL, Botteron K, Repovs G, Barch DM: Default mode network connectivity in children with a history of preschool onset depression. J Child Psychol Psychiatry 2012;53:964-972.

23 Smith SM, Beckmann CF, Andersson J, Auerbach EJ, Bijsterbosch J, Douaud G, Duff E, Feinberg DA, Griffanti L, Harms MP, Kelly M, Laumann T, Miller KL, Moeller S, Petersen S, Power J, Salimi-Khorshidi G, Snyder AZ, Vu AT, Woolrich MW, Xu J, Yacoub E, Ugurbil K, Van Essen DC, Glasser MF; WU-Minn HCP Consortium: Resting-state fMRI in the Human Connectome Project. Neuroimage 2013;80:144-168.

24 Sylvester CM, Barch DM, Corbetta M, Power JD, Schlaggar BL, Luby JL: Resting state functional connectivity of the ventral attention network in children with a history of depression or anxiety. J Am Acad Child Adolesc Psychiatry 2013;52:1326-1336.

25 Fox MD, Snyder AZ, Vincent JL, Corbetta M, Van Essen DC, Raichle ME: The human brain is intrinsically organized into dynamic, anticorrelated functional networks. Proc Natl Acad Sci USA 2005;102:9673-9678. 
26 Vincent JL, Snyder AZ, Fox MD, Shannon BJ, Andrews JR, Raichle ME, Buckner RL: Coherent spontaneous activity identifies a hippocampal-parietal memory network. J Neurophysiol 2006;96:3517-3531.

27 Vincent JL, Kahn I, Snyder AZ, Raichle ME, Buckner RL: Evidence for a frontoparietal control system revealed by intrinsic functional connectivity. J Neurophysiol 2008;100: 3328-3342.

28 Talairach J, Tournoux P: Co-Planar Stereotaxic Atlas of the Human Brain. New York, Thieme Medical, 1988.

29 Talairach J, Tournoux P, Missir O: Referentially Oriented Cerebral MRI Anatomy: An Atlas of Stereotaxic Anatomical Correlations for Gray and White Matter. New York, Thieme Medical, 1993.

30 Lancaster JL, Glass TG, Lankipalli BR, Downs H, Mayberg H, Fox PT: A modality-independent approach to spatial normalization of tomographic images of the human brain. Hum Brain Mapp 1995;3:209-223.

- 31 Power JD, Barnes KA, Snyder AZ, Schlaggar BL, Petersen SE: Spurious but systematic correlations in functional connectivity MRI networks arise from subject motion. Neuroimage 2012;59:2142-2154.

- 32 Friston KJ, Williams S, Howard R, Frackowiak RS, Turner R: Movement-related effects in fMRI time-series. Magn Reson Med 1996;35: 346-355.

-33 Cordes D, Haughton VM, Arfanakis K, Carew JD, Turski PA, Moritz CH, Quigley MA, Meyerand ME: Frequencies contributing to functional connectivity in the cerebral cortex in 'resting-state' data. AJNR Am J Neuroradiol 2001;22:1326-1333.

- 34 Birn RM, Diamond JB, Smith MA, Bandettini PA: Separating respiratory-variation-related fluctuations from neuronal-activity-related fluctuations in fMRI. Neuroimage 2006;31: 1536-1548.
5 Smyser CD, Inder TE, Shimony JS, Hill JE, Degnan AJ, Snyder AZ, Neil JJ: Longitudinal analysis of neural network development in preterm infants. Cereb Cortex 2010;20:28522862.

36 Power JD, Petersen SE: Control-related systems in the human brain. Curr Opin Neurobiol 2013;23:223-228.

37 Satterthwaite TD, Wolf DH, Ruparel K, Erus G, Elliott MA, Eickhoff SB, Gennatas ED, Jackson C, Prabhakaran K, Smith A, Hakonarson H, Verma R, Davatzikos C, Gur RE, Gur RC: Heterogeneous impact of motion on fundamental patterns of developmental changes in functional connectivity during youth. Neuroimage 2013;83:45-57.

38 Power JD, Cohen AL, Nelson SM, Wig GS, Barnes KA, Church JA, Vogel AC, Laumann TO, Miezin FM, Schlaggar BL, Petersen SE: Functional network organization of the human brain. Neuron 2011;72:665-678.

39 Power JD, Mitra A, Laumann TO, Snyder AZ, Schlaggar BL, Petersen SE: Methods to detect, characterize, and remove motion artifact in resting state fMRI. Neuroimage 2014;84:320 341.

40 Power JD, Schlaggar BL, Petersen SE: Recent progress and outstanding issues in motion correction in resting state fMRI. Neuroimage 2015;105C:536-551.

41 Fisher RA: Frequency distribution of the values of the correlation coefficient in samples from an indefinitely large population. Biometrika 2014;10:507-521.

42 Rubens FD, Boodhwani M, Nathan H: Interpreting studies of cognitive function following cardiac surgery: a guide for surgical teams. Perfusion 2007;22:185-192.
43 Inagaki M, Yoshikawa E, Matsuoka Y, Sugawara $\mathrm{Y}$, Nakano T, Akechi T, Wada N, Imoto S, Murakami K, Uchitomi Y: Smaller regional volumes of brain gray and white matter demonstrated in breast cancer survivors exposed to adjuvant chemotherapy. Cancer 2007;109: 146-156.

-44 Silverman DH, Dy CJ, Castellon SA, Lai J, Pio BS, Abraham L, Waddell K, Petersen L, Phelps ME, Ganz PA: Altered frontocortical, cerebellar, and basal ganglia activity in adjuvanttreated breast cancer survivors 5-10 years after chemotherapy. Breast Cancer Res Treat 2007;103:303-311.

45 De Ruiter MB, Reneman L, Boogerd W, Veltman DJ, Caan M, Douaud G, Lavini C, Linn SC, Boven E, van Dam FS, Schagen SB: Late effects of high-dose adjuvant chemotherapy on white and gray matter in breast cancer survivors: converging results from multimodal magnetic resonance imaging. Hum Brain Mapp 2012;33:2971-2983.

46 Deprez S, Amant F, Yigit R, Porke K, Verhoeven J, Van den Stock J, Smeets A, Christiaens MR, Leemans A, Van Hecke W, Vandenberghe J, Vandenbulcke M, Sunaert S: Chemotherapy-induced structural changes in cerebral white matter and its correlation with impaired cognitive functioning in breast cancer patients. Hum Brain Mapp 2011;32:480-493.

47 Deprez S, Amant F, Smeets A, Peeters R, Leemans A, Van Hecke W, Verhoeven JS, Christiaens MR, Vandenberghe J, Vandenbulcke $\mathrm{M}$, Sunaert S: Longitudinal assessment of chemotherapy-induced structural changes in cerebral white matter and its correlation with impaired cognitive functioning. J Clin Oncol 2012;30:274-281.

-48 Hosseini SM, Kesler SR: Multivariate pattern analysis of fMRI in breast cancer survivors and healthy women. J Int Neuropsychol Soc 2014;20:391-401. 